
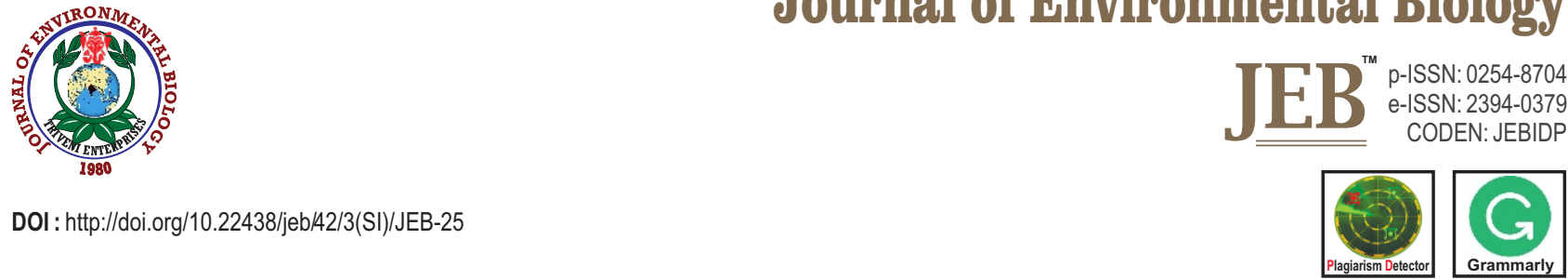

\title{
Species composition and biodiversity indexes of weeds in paddy rice on two sides of dike system during three crops in an Giang Province, Vietnam
}

\author{
M.D. Quan ${ }^{1 *}$, T.T. Hang ${ }^{2}$ and S.T. Nam ${ }^{3}$ \\ ${ }^{1}$ Department of Biology, School of Education, Can Tho University, Can Tho, 900000, Vietnam \\ ${ }^{2}$ Le Hoai Don High School, Ben Tre 930000, Vietnam \\ ${ }^{3}$ College of Environment and Natural Resources, Can Tho University, Can Tho, 900000, Vietnam \\ *Corresponding Author Email : dmquan@ctu.edu.vn
}

\section{Abstract}

Aim: This study was conducted to provide data on weed species composition and biodiversity indexes at different rice crops inside and outside of the dike system in An Giang province, Vietnam.

Methodology: Weeds were collected from paddy rice of inner and outer side of dike system from six districts of An Giang province, Vietnam. From each sampling sites, two quadrats with $100 \mathrm{~m}^{2}(10 \mathrm{~m} \times 10 \mathrm{~m})$ per quadrat were selected to collect weed after $75-80$ days of rice planting. Weeds were collected and identified for their scientific name, and the number of each species was defined, followed by calculation of the occurrence frequency and density of samples. Biodiversity indices viz. Shannon-Weaver $\left(H^{\prime}\right)$, Simpson (1- $\left.\lambda\right)$, Margalef $(d)$, and similarity index were also studied among three crops and two sides of dike system.

Results: The weed composition in the paddy rice in An Giang was diversity with 137 species. Among them, the most species belonged to families Poaceae, Cyperaceae, and Compositae. The species composition of Crop 3 was the highest one. Species occurrence frequency and weed density of groups C, D, E reached the highest value in Crop 1, followed by Crop 2 and 3 . The biodiversity indices of weed species composition in the studied sites were high, reaching the highest value in Crop 3.

Interpretation: The results of this study enhance the knowledge of weed species composition and biodiversity indexes to effectively manage and control weeds in rice cultivation.

Key words: Biodiversity index, Dike system, Paddy rice, Weeds

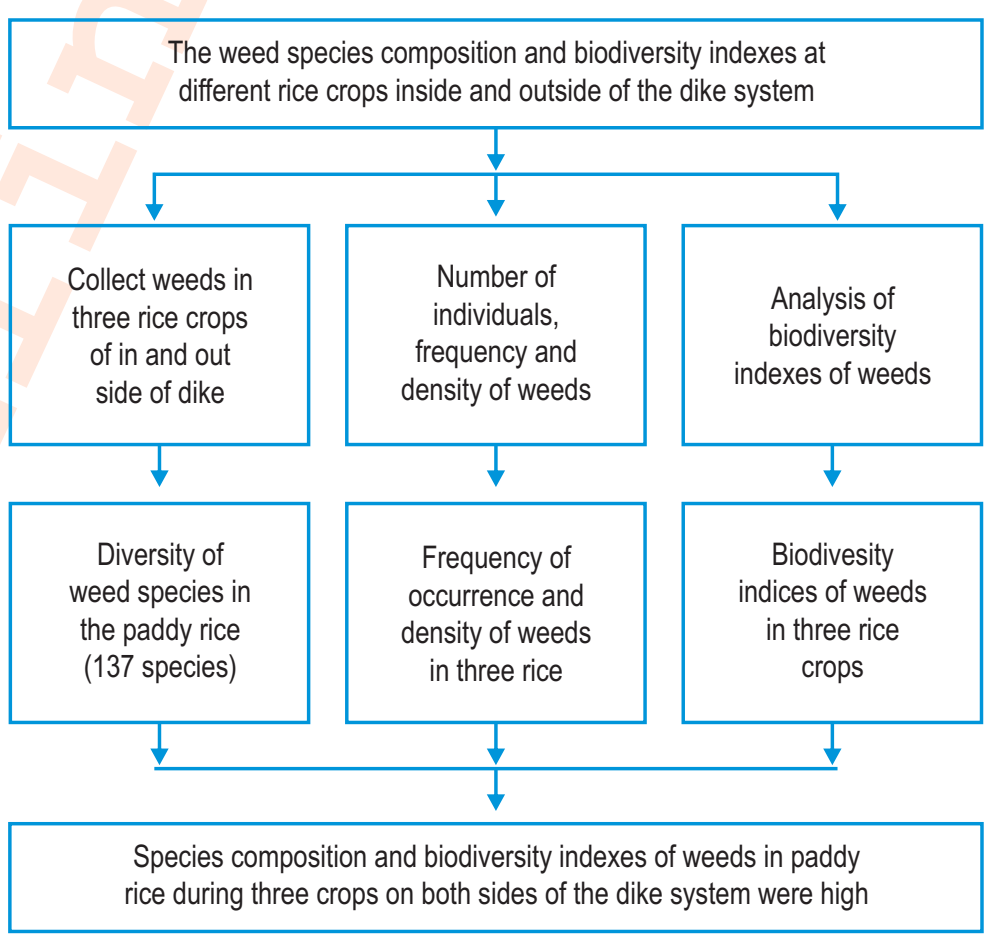

How to cite : Quan, M.D., T.T. Hang and S.T. Nam: Species composition and biodiversity indexes of weeds in paddy rice on two sides of dike system during three crops in an Giang Province, Vietnam. J. Environ. Biol., 42, 895-902 (2021). 


\section{Introduction}

The Mekong Delta located in the south part of Vietnam,where climate conditions and condense river systems facilitatefor the development of plants, rice crops especially. In theMekong Delta, the plant composition is diverse in rice paddyfields, as shown by 188 identified species (Duong et al., 2003). These species influence the productivity and quality of rice, for example, they compete with rice for light, nutrition, and water. Moreover, they support the growth of pests and plant diseases and provide shelters for rice-destroying rats. However, many of these species are beneficial for local people as they can be used as vegetables, traditional medicine, and natural fertilizer.

An Giang province is situated across Tien and Hau rivers, which belong to the Mekong river system. This province has enriched surface water and a high sediment level, which facilitates the growth of rice. Interestingly, most of An Giang areas are covered with flood water 1-4 m deep from the end of July to early December every year (Duong et al., 2017). Therefore, prior to 1995 , rice was annually produced by two rice crops whichproduce 5.25 tons ha (Huynh et al., 2013). To increase cropping areas and rice productivity, as well as to control flood impacts, a dike system was built at multiple areas to additionally produce triple rice cropping (Nguyen et al., 2016). As a result, it has been formed three-rice-crop regions (fulldyke system) different with two-rice crops areas (semi-dykesystem). Although the dike system provides some benefits for the growth of rice, it caused several drawbacks such as preventing sediment flow to rice fields, and water exchange between rice fields and rivers (Pham et al., 2015), affecting the local ecosystem and biological diversity. There are significant differences between the species composition from Outer dike and Inner dike (Dinh et al., 2020). However, since the dike system has been build, the plant composition of rice fields areas has not examined. Therefore, this study aimed to provide data on the weed species composition and biodiversity indices at different rice crops inside and outside of the dike system in An Giang province, Vietnam.

\section{Materials and Methods}

This study was conducted during three crops: Crop 1 is from 4/2019 to 7/2019, Crop 2 is from 8/2019 to 11/2019, and Crop 3 is from 12/2019 to 3/2020. Samples were collected within $75-80$ days after the rice was planted. This time was selected because of the mature weeds, which aid in the classification, collection and analysis of data samples.

The study area included six districts Cho Moi, Chau Phu, Tinh Bien, Tri Ton, Thoai Son, and Chau Doc in An Giang province. At each district, two rice fields examined: Outer dike (two crops per year) and Inner dike (three crops per year) (Fig. 1).

To survey local farmers and agricultural managers in An Giang, the method of Praticipatory Rural Appraisal (Nguyen and
Vromant, 2009) was used. This method helps to generate data of rice-growing sites, crops, and plant composition within rice fields.

To investigate sampling sites and to design quadrats of sampling, methods of Nguyen (2007) were applied. At each sampling sites, there was two standard sampling plots (quadrat) located $150-200 \mathrm{~m}$ apart, with $100 \mathrm{~m}^{2}(10 \mathrm{~m} \times 10 \mathrm{~m})$ per quadrat. The quadrates were placed through three field habitats, including inside, banks of rice field and along the small canals that carry water into the rice field. There were 5 sub-squares of sample collection, each had $1 \mathrm{~m}^{2}$ at 4 corners and central of the quadrat. Plants were collected and identified for their scientific name before calculating the occurrence frequency and density of samples.

The scientific names of plant samples were identified byusing taxonomic comparison, in combination with usingreferences from published data of plant classification (Duong et al., 2003; Pham, 1999; Pham, 2000; Pham, 2003; Suk et al., 2000; Tran et al., 2018; Vo, 2002; Vo, 2004). The plant list thenwas adjustified according to Checklist of Plant species ofVietnam (Nguyen and Dorofeev, 2003; Nguyen and Dorofeev, 2005) and information from the website of The Plant List (2013).

The occurrence frequency of plant species was calculated by the formula: $F(\%)=p i / p \times 100$ ( $p_{i}$ : number of quadrats in which the species occurs, $P$ : total number of quadrats examined) (Sharma, 2003). Based on $F$ values, plants were arranged into 5 groups: Group A with very low occurrence frequency $(F=1-20 \%)$; Group $B$ with low occurrence frequency $(\mathrm{F}=21-40 \%)$; Group C with moderate occurrence frequency $(\mathrm{F}=41-60 \%)$; Group $\mathrm{D}$ with high occurrence frequency ( $\mathrm{F}=61$ $80 \%$ ) and group $E$ with very high occurrence frequency ( $F=81$ $100 \%)$.

The density (individuals $\mathrm{m}^{-2}$ ) was calculated: D $=$ (individual $\mathrm{m}^{-2}$ ) $=$ Total number of individuals of a species in allthe quadrats / Number of quadrats examined (Sharma, 2003).

Biodiversity indices such as Shannon-Weaver's diverse index: (Shannon and Weaver, 1949), Simpson's dominant index: I (Simpson, 1949), Margalef's abundant index: $d=$ (Margalef, 1958) $\left(p_{i}=n_{i} / N, n_{i}\right.$ : the number of individuals of $i^{\text {th }}$ species; $S$ : the number of species; $\mathrm{N}$ : total number of individuals of all species in all quarats examined) were estimated by PRIMER v. 6 (Clarke and Gorley, 2006). The S17 Bray Curtis Similarity Index was used to determine the similarity of weed species composition among three crops and two sides of dike system (Bray and Curtis, 1957).

The fluctuation of plant composition toward crops and dike features was examined by PERMANOVA test. The correlation between several factors, including crop $x$ site of sampling in the crop $x$ dike, sampling site $x$ dike; and plant composition was examined by PERMANOVA test. The PRIMER 


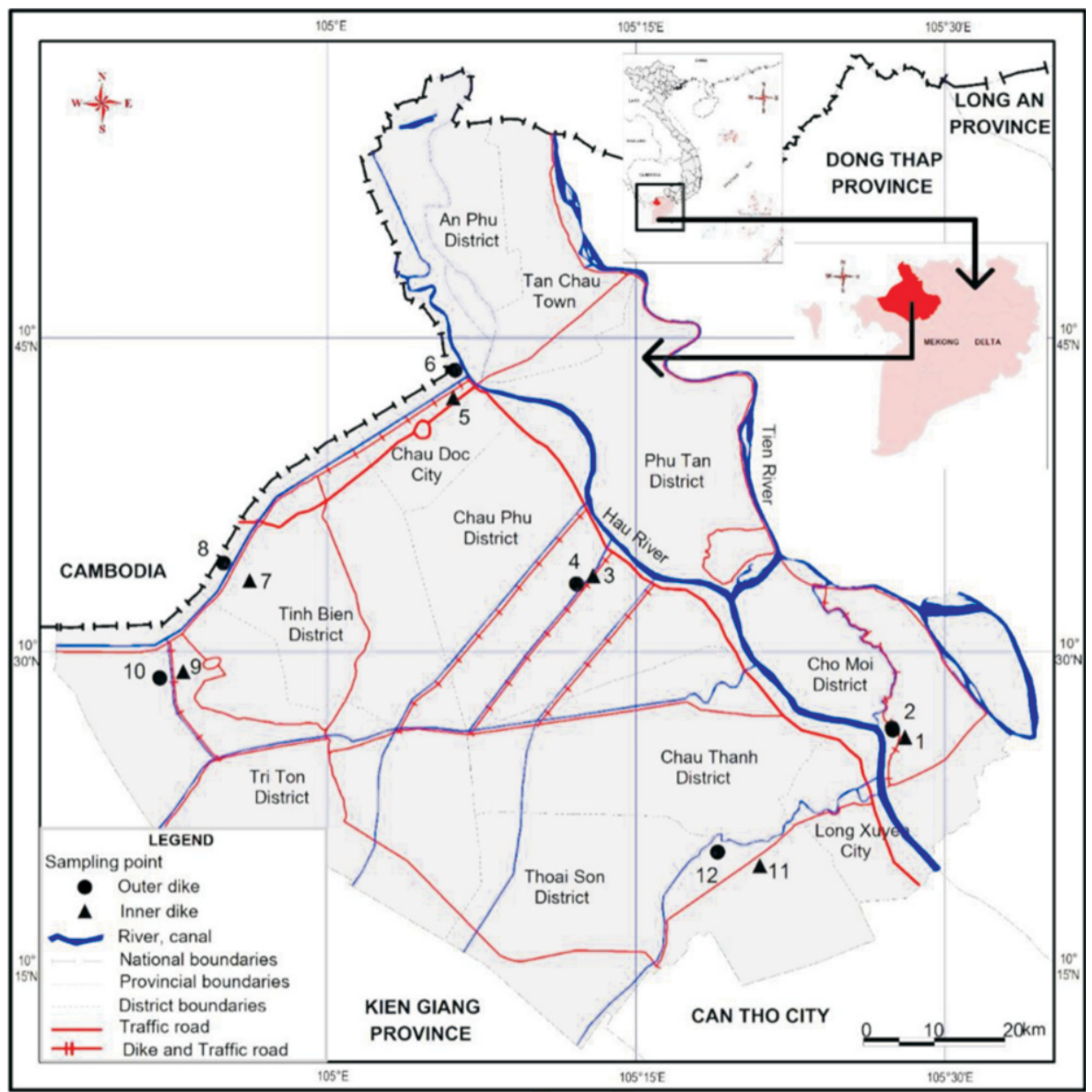

Fig. 1 : Illustration of sampling sites of rice crop in An Giang province.

v6 software was used in combination with PERMANOVA for data analysis. All tests were performed with $p$-value of $5 \%$.

\section{Results and Discussion}

In this study, 478 weed samples were collected from 12 rice-growing sites (in 6 districts in An Giang province). They were stored at the Plant Laboratory at Can Tho University, Can Tho city, Vietnam. Based on the classification result, 137 species belonged to 103 genera of 42 families in 2 phyla, Polypodiophyta and Magnoliophyta, were identified. Of which, Magnoliophyta was dominant with 135 species belonging to 101 genera of 40 families, Polypodiophyta had only 2 species belonging to 2 genera of 2 families. Three families with the highest number of species were Poaceae with 24 species, Cyperaceae with 14 species, and Compositae with 10 species. Compositae species Sphaeranthus africanus, Eclipta prostrata, Vernonia cinerea were distributed mainly along the coast and on the banks of rice field such. Species of families Poaceae and Cyperaceae were distributed both onshore, onshore and in flooded fields, typically
Echinochloa crus-galli, Leptochloa chinensis, Oryza rufipogon (Poaceae) and Cyperus digitatus, Cyperus iria, Eleocharis dulcis, Fimbristylis quinquangularis (Cyperaceae). Four genera with the highest number of species were Cyperus with 8 species, Paspalum with 5 species, Ludwigia and Euphorbia with 4 species. Compared to the results of Duong et al. (2003) with 75 species, 43 genera and 30 plant families were found in wet rice fields in Vietnam. This study showed more diverse taxon composition, since this was conducted in rice fields, near the field and on the edge of the field, therefore, the species composition had both aquatic and terrestrial weeds. However, this result was also similar to previous result (Duong et al., 2003) which dominated in the number of species of the Poaceae and Cyperaceae.

The most diverse weed species were found in Crop 3 with 111 species accounting for $81.02 \%$ of the total number of species, followed by Crop 1 with 101 species (accounting for $73.72 \%$ of the total number of species), and the lowest was Crop 2 with 62 species (accounting for $45.26 \%$ of the total number of species). The Crop 2 is the flooding season, therefore, the survey was only 
Table 1 : Frequency of occurrence and density of weed species collected in rice growing areas in An Giang province

\begin{tabular}{|c|c|c|c|c|c|c|c|c|c|c|c|c|c|}
\hline \multirow[t]{2}{*}{ Scientific name } & \multicolumn{5}{|c|}{ Occurrence frequency (\%) } & \multicolumn{5}{|c|}{ Density (individual $\mathrm{m}^{-2}$ ) } & \multicolumn{3}{|c|}{ Biodiversity indices } \\
\hline & $\mathrm{C} 1$ & $\mathrm{C} 2$ & C3 & $\ln$ & Out & $\mathrm{C} 1$ & $\mathrm{C} 2$ & C3 & In & Out & $d$ & $H^{\prime}$ & $1-e^{\prime}$ \\
\hline quadrifolia L. & 8.30 & 0.00 & 8.30 & 0 & 16.70 & 042 & 0.000 & 0.017 & 000 & 0.058 & 9854 & 0.5637 & 0.7476 \\
\hline Ceratopteris thalictroides (L.) Brongn. & 25.00 & 16.70 & 25.00 & 16.70 & 33.30 & 042 & 0.050 & 0.050 & 0.033 & 0.067 & 1.8728 & 0.8679 & 0.8769 \\
\hline Ruellia tuberosa L. & 25.00 & 16.70 & 16.70 & 3.30 & 0.00 & 0.042 & 0.017 & 0.025 & 0.050 & 0.000 & 2.1239 & 0.7970 & 0.8376 \\
\hline chyranthes aspera L. & 0.00 & 16.70 & 8.30 & 11.10 & 0.00 & 0.000 & 0.033 & 0.017 & 0.022 & 0.000 & 1.6097 & 0.6778 & 0.8485 \\
\hline Alternanthera paronychioides St.-Hil. & 3.30 & 66.70 & 33.30 & 61.10 & 58.30 & .217 & 0.200 & 0.108 & 0.172 & 0.167 & 1.9879 & 0.9610 & \\
\hline Alternanthera & 16.70 & 0.00 & 16.70 & 0.00 & 33.30 & .025 & 0.000 & 0.017 & 0.000 & 0.042 & 1.8463 & 0.7273 & 0.8476 \\
\hline Amaranthus lividus L. & 16.70 & 0.00 & 8.30 & 0.00 & 25.00 & .025 & 0.000 & 0.008 & 0.000 & 0.033 & 1.6097 & 0.6589 & 0.8333 \\
\hline Amaranthus spinosus L. & 8.30 & 0.00 & 8.30 & 0.00 & 16.70 & .008 & 0.000 & 0.017 & 0.000 & 0.025 & 1.8205 & 0.6614 & \\
\hline thus tricolor L. & 30 & 0.00 & 0.00 & 0.00 & 8.30 & .008 & 0.000 & 0.000 & 0.000 & 0.008 & 1.8205 & 0.4771 & 1.0000 \\
\hline osioide & 30 & 0.00 & 8.30 & 11.10 & 0.00 & .017 & 0.000 & 0.008 & & 0.000 & & 693 & 56 \\
\hline Cente & 8.30 & 0.00 & 0.00 & 0.00 & 8.30 & 0.017 & 0.000 & 0.000 & 0.000 & 0.017 & 1.1162 & 0.4771 & 0.8000 \\
\hline Ageratu & 0.00 & 0.00 & 8.30 & 22.20 & 25.00 & 050 & 0.000 & 0.008 & 0.022 & 0.025 & 2.2992 & 202 & 0.8667 \\
\hline Eclipt & 58.30 & 16.70 & 33.30 & 38.90 & 41.70 & 058 & 0.083 & 0.067 & 83 & 0.042 & 2.4424 & 79 & \\
\hline Emi & 00 & 16.70 & 8.30 & 10 & 0.00 & 000 & 0.017 & 0.008 & & 0.000 & 2.2324 & 78 & \\
\hline & 8.30 & 0.00 & 8.30 & 11.10 & 0.00 & 008 & 0.000 & 0.008 & 11 & 0.000 & 2.2324 & 778 & \\
\hline Praxelis clematidea (C & .00 & 16.70 & 8.30 & 16.70 & 16.70 & 058 & 0.033 & 0.017 & 33 & 0.042 & 2.0020 & 82 & \\
\hline \multicolumn{14}{|l|}{ R.M.King \& H.Rob. } \\
\hline thus africa & 0 & 0.00 & 1 & 0 & 16.70 & 00 & 00 & 25 & & 0.025 & & & \\
\hline & 0.00 & 16.70 & 16.70 & 11.10 & 8.30 & .000 & 0.033 & 0.025 & 22 & 0.008 & 2.2156 & & \\
\hline & 25.00 & 0.00 & 0.00 & 1.10 & 8.30 & .033 & 0.000 & 0.000 & 0.017 & 0.008 & 2.0121 & & \\
\hline $7 s \mathrm{~L}$. & 0.00 & 16.70 & 16.70 & 6.70 & 0.00 & .000 & 0.017 & 0.025 & 0.022 & 0.000 & 1.6097 & 99 & 82 \\
\hline & 16.70 & 0.00 & 16.70 & 1.10 & 16.70 & 025 & 0.000 & 0.017 & 11 & 0.025 & 2.2156 & & \\
\hline & 8.30 & 0.00 & 8.30 & 0.00 & 16.70 & .008 & 0.000 & 0.017 & 0.000 & 0.025 & & & \\
\hline & 8.30 & 0.00 & 8.30 & 0.00 & 16.70 & 0.017 & 0.000 & 0.008 & 0.000 & 0.025 & 1.8205 & & \\
\hline$u m \mathrm{~L}$. & 25.00 & 16.70 & 25.00 & 16.70 & 33.30 & 0.050 & 0.017 & 0.042 & 0.028 & 0.058 & 2.2 & 20 & \\
\hline iiiL.f. & 41.70 & 0.00 & 16.70 & 6.70 & 33.30 & 050 & 0.000 & 0.033 & 0.028 & 0.042 & & 27 & \\
\hline & 00 & 16.70 & 0.00 & 5.60 & 0.00 & 00 & 0.033 & 0.000 & 0.011 & 0.000 & & 71 & 000 \\
\hline 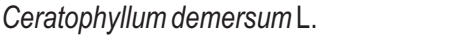 & 0.00 & 16.70 & 8.30 & 5.60 & 8.30 & 0.000 & 0.033 & 0.008 & 0.011 & 0.008 & & & \\
\hline Ip & 41.70 & 16.70 & 33.30 & 27.80 & 41.70 & 0.058 & 0.017 & 0.058 & 0.039 & 0.067 & 2.6 & & \\
\hline & 16.70 & 0.00 & 16.70 & 10 & 16.70 & 17 & 0.000 & 025 & 17 & 0.017 & & & \\
\hline erf. & 8.30 & 16.70 & 25.00 & .10 & 25.00 & 008 & 0.050 & 0.042 & 28 & 0.033 & & & 332 \\
\hline & 8.30 & 16.70 & 8.30 & & 8.30 & & 0.017 & & & 0.008 & & & \\
\hline Coc & 0.00 & 16.70 & 8.30 & 11.10 & 0.00 & 00 & 0.017 & 0.008 & 0.011 & 0.000 & & & \\
\hline lum cochinct & 0.00 & & 8.30 & 11.10 & 0.00 & 000 & 0.017 & 0.008 & 11 & 0.000 & 2.2324 & 78 & 333 \\
\hline & 0.00 & 0.00 & & 0.00 & 8.30 & & & & & 0.008 & & & \\
\hline & 0.00 & 16.70 & 0.00 & 5.60 & 0.00 & 000 & 0.017 & 0.000 & 0.006 & 0.000 & & & \\
\hline . & 0.00 & 0.00 & & 5.60 & 0.00 & .000 & 0.000 & 0.017 & & 0.000 & & & \\
\hline & 8.30 & 0.00 & & 0.00 & 8.30 & .017 & 0.000 & 0.000 & 0.000 & 0.017 & & 62 & \\
\hline ophylla L. & 8.30 & 33.30 & 16.70 & 27.80 & 0.00 & 0.008 & 0.033 & 0.025 & 0.033 & 0.000 & & 0.6723 & 0.8000 \\
\hline & 25.00 & 16.70 & 0.00 & 11.10 & 16.70 & 0.025 & 0.017 & 0.000 & 0.011 & 0.017 & 2.5022 & 0.8225 & 0.9273 \\
\hline & 8.30 & 0.00 & 16.70 & 11.10 & 8.30 & 0.008 & 0.000 & 0.017 & 0.011 & 0.008 & 2.7307 & 0.8205 & \\
\hline olia L. & 8.30 & 0.00 & 8.30 & 0.00 & 16.70 & .017 & 0.000 & 0.008 & 0.000 & 0.025 & & 0.5693 & 0.8056 \\
\hline Phy & 8.30 & 0.00 & 16.70 & 0.00 & 25.00 & 0.008 & 0.000 & 0.017 & 0.000 & 0.025 & 2.4045 & 0.7242 & 0.8929 \\
\hline latus Poir. & 8.30 & 0.00 & 8.30 & 0.00 & 16.70 & 0.008 & 0.000 & 0.008 & 0.000 & 0.017 & 1.3464 & 0.5162 & 0.5951 \\
\hline & 75.00 & 33.30 & 33.30 & 44.40 & 58.30 & 0.208 & 0.083 & 0.058 & 0.111 & 0.142 & 2.0782 & 0.8396 & 0.8379 \\
\hline & 8.30 & 0.00 & 8.30 & 11.10 & 0.00 & 0.008 & 0.000 & 0.008 & 0.011 & 0.000 & & 0.6773 & 0.8929 \\
\hline um triflorum & 0.00 & 16.70 & 8.30 & 5.60 & 8.30 & 0.000 & 0.033 & 0.017 & 0.011 & 0.017 & 1.5157 & 0.6836 & 0.8462 \\
\hline Macroptilium lathyroides (L.) Urb. & 16.70 & 33.30 & 0.00 & 16.70 & 8.30 & 0.025 & 0.050 & 0.000 & 0.028 & 0.008 & 2.1177 & 0.7636 & 0.8529 \\
\hline & & & & & & & & & & 033 & & & \\
\hline Vigna adenantha (F. G. Mey.) & 25.00 & 0.00 & 0.00 & 5.60 & 16.70 & 0.025 & 0.000 & 0.000 & 0.006 & 0.017 & 2.1747 & 0.7186 & 0.7700 \\
\hline
\end{tabular}
Masch. \& Stain. 
Basilicum polystachyon (L.) Moench Hyptis rhomboidea Mart. \& Gal. Hyptis suaveolens (L.) Poit. Ocimum gratissimum L. Utricularia aurea Lour. Sida rhombifolia L. Urena lobata L.

Nymphoidesindicum (L.) O. Kuntze Mimosa pigra L.

Neptunia oleracea Lour. Glinus oppositifolius (L.) DC. Nelumbo nucifera Gaertn. Nymphaea nouchaliBurm. f. Nymphaea pubescens Willd. Nymphaea rubra Roxb. ex Salisb. Ludwigia adscendens (L.) Hara Ludwigia hyssopifolia (G. Don) Exell apudA. \& R. Fernandes Ludwigia octovalvis (Jacq.) Raven Ludwigia prostrata Roxb. Passiflora foetida L.

Persicaria pulchra (Blume) Sojak Portulaca oleracea L. Dentella repens (L.) Forst. \& Forst. f. Oldenlandia corymbosa L.

Oldenlandia diffusa (Willd.) Roxb. Morinda persicaefolia Buch.-Ham. Cardiospermum halicacabum L. Bacopa monnieri (L.) Wettst. Lindernia crustacea (L.) F. Muell. Lindernia antipoda (L.) Alst. Scoparia dulcis L.

Physalis angulata L.

Sphenoclea zeylanica Gaertn. Melochia corchorifolia L. Pentapetes phoenicea L. Pilea microphylla (L.) Liebm. Pouzolzia zeylanica(L.) Liebm. Cayratia trifolia (L.) Domin Colocasia esculenta (L.) Schott Cryptocoryne ciliata (Roxb.)

Fisch ex Schott

Lemna perpusilla Torr.

Pistia stratiotes L.

Typhonium flagelliforme (Lodd.) Blume Commelina diffusa Burm. $f$.

Cyperus babakan Steud.

Cyperus difformis L.

Cyperus digitatus Roxb.

Cyperus elatus L.

Cyperushalpan L.

Cyperus imbricatus Retz.

CyperusiriaL.

Cypeus rotundus L.

Eleocharis dulcis (Burm. f.) Hensch Fimbristylis aestivalis (Retz.) Vahl Fimbristylis quinquangularis (Vahl) Kunth Kyllinga brevifolia Rottb. $\begin{array}{lllllllllllll}33.30 & 33.30 & 25.00 & 33.30 & 25.00 & 0.042 & 0.183 & 0.025 & 0.089 & 0.025 & 1.6265 & 0.6135 & 0.7051\end{array}$ $\begin{array}{lllllllllllll}0.00 & 0.00 & 8.30 & 0.00 & 8.30 & 0.000 & 0.000 & 0.017 & 0.000 & 0.017 & 1.4427 & 0.6021 & 0.8571\end{array}$ $\begin{array}{lllllllllllll}8.30 & 16.70 & 0.00 & 11.10 & 0.00 & 0.017 & 0.033 & 0.000 & 0.022 & 0.000 & 0.9102 & 0.4191 & 0.6667\end{array}$ $\begin{array}{lllllllllllll}0.00 & 0.00 & 8.30 & 5.60 & 0.00 & 0.000 & 0.000 & 0.008 & 0.006 & 0.000 & 1.5595 & 0.6135 & 0.7821\end{array}$ $\begin{array}{lllllllllllll}8.30 & 16.70 & 8.30 & 11.10 & 8.30 & 0.042 & 0.033 & 0.033 & 0.039 & 0.033 & 0.9208 & 0.5616 & 0.7292\end{array}$ $\begin{array}{lllllllllllll}0.00 & 0.00 & 33.30 & 5.60 & 25.00 & 0.000 & 0.000 & 0.033 & 0.006 & 0.025 & 2.9192 & 0.8565 & 0.9273\end{array}$ $\begin{array}{lllllllllllll}0.00 & 16.70 & 8.30 & 11.10 & 0.00 & 0.000 & 0.017 & 0.017 & 0.017 & 0.000 & 1.4427 & 0.5737 & 0.8214\end{array}$ $\begin{array}{lllllllllllll}0.00 & 0.00 & 8.30 & 0.00 & 8.30 & 0.000 & 0.000 & 0.017 & 0.000 & 0.017 & 1.8205 & 0.6867 & 0.8889\end{array}$ $\begin{array}{lllllllllllll}16.70 & 16.70 & 8.30 & 16.70 & 8.30 & 0.017 & 0.017 & 0.017 & 0.022 & 0.008 & 2.4218 & 0.7952 & 0.8497\end{array}$ $\begin{array}{lllllllllllll}8.30 & 0.00 & 16.70 & 0.00 & 25.00 & 0.017 & 0.000 & 0.050 & 0.000 & 0.067 & 0.9854 & 0.5772 & 0.7571\end{array}$ $\begin{array}{lllllllllllll}0.00 & 0.00 & 8.30 & 0.00 & 8.30 & 0.000 & 0.000 & 0.042 & 0.000 & 0.042 & 0.7059 & 0.4713 & 0.6985\end{array}$ $\begin{array}{lllllllllllll}0.00 & 0.00 & 25.00 & 5.60 & 16.70 & 0.000 & 0.000 & 0.058 & 0.011 & 0.042 & 1.7648 & 0.7502 & 0.8603\end{array}$ $\begin{array}{lllllllllllll}0.00 & 16.70 & 0.00 & 5.60 & 0.00 & 0.000 & 0.050 & 0.000 & 0.017 & 0.000 & 0.8341 & 0.4634 & 0.7091\end{array}$ $\begin{array}{lllllllllllll}8.30 & 0.00 & 0.00 & 5.60 & 0.00 & 0.042 & 0.000 & 0.000 & 0.028 & 0.000 & 1.1368 & 0.5609 & 0.7582\end{array}$ $\begin{array}{lllllllllllll}8.30 & 0.00 & 8.30 & 5.60 & 8.30 & 0.017 & 0.000 & 0.017 & 0.011 & 0.017 & 1.5533 & 0.6896 & 0.7867\end{array}$ $\begin{array}{lllllllllllll}25.00 & 16.70 & 8.30 & 11.10 & 25.00 & 0.042 & 0.167 & 0.017 & 0.067 & 0.042 & 1.3677 & 0.7101 & 0.7688\end{array}$ $\begin{array}{lllllllllllll}91.70 & 83.30 & 58.30 & 66.70 & 83.30 & 0.467 & 0.783 & 0.250 & 0.433 & 0.458 & 1.7355 & 0.9236 & 0.8605\end{array}$

$\begin{array}{lllllllllllll}58.30 & 33.30 & 33.30 & 27.80 & 66.70 & 0.217 & 0.250 & 0.092 & 0.178 & 0.167 & 1.5972 & 0.8456 & 0.8348\end{array}$ $\begin{array}{lllllllllllll}83.30 & 50.00 & 66.70 & 61.10 & 83.30 & 0.633 & 0.750 & 0.458 & 0.478 & 0.750 & 1.3630 & 0.7406 & 0.7836\end{array}$ $\begin{array}{lllllllllllll}0.00 & 0.00 & 16.70 & 5.60 & 8.30 & 0.000 & 0.000 & 0.017 & 0.006 & 0.008 & 2.2324 & 0.6778 & 0.9333\end{array}$ $\begin{array}{lllllllllllll}0.00 & 0.00 & 8.30 & 5.60 & 0.00 & 0.000 & 0.000 & 0.017 & 0.011 & 0.000 & 1.6097 & 0.6374 & 0.8030\end{array}$ $\begin{array}{lllllllllllll}25.00 & 16.70 & 8.30 & 22.20 & 8.30 & 0.042 & 0.017 & 0.017 & 0.039 & 0.008 & 1.6981 & 0.6583 & 0.7778\end{array}$ $\begin{array}{lllllllllllll}8.30 & 0.00 & 16.70 & 11.10 & 8.30 & 0.008 & 0.000 & 0.017 & 0.011 & 0.008 & 1.4270 & 0.5613 & 0.6950\end{array}$ $\begin{array}{lllllllllllll}50.00 & 50.00 & 25.00 & 44.40 & 33.30 & 0.358 & 0.600 & 0.417 & 0.400 & 0.475 & 1.5798 & 0.8921 & 0.8546\end{array}$ $\begin{array}{lllllllllllll}41.70 & 33.30 & 33.30 & 22.20 & 58.30 & 0.142 & 0.183 & 0.100 & 0.111 & 0.167 & 1.3654 & 0.7338 & 0.8090\end{array}$ $\begin{array}{lllllllllllll}8.30 & 0.00 & 0.00 & 5.60 & 0.00 & 0.008 & 0.000 & 0.000 & 0.006 & 0.000 & 1.8205 & 0.4771 & 1.0000\end{array}$ $\begin{array}{lllllllllllll}0.00 & 0.00 & 8.30 & 5.60 & 0.00 & 0.000 & 0.000 & 0.008 & 0.006 & 0.000 & 1.3029 & 0.5074 & 0.7111\end{array}$ $\begin{array}{llllllllllllll}16.70 & 0.00 & 8.30 & 5.60 & 16.70 & 0.042 & 0.000 & 0.025 & 0.017 & 0.042 & 1.5533 & 0.7677 & 0.8600\end{array}$ $\begin{array}{lllllllllllll}16.70 & 0.00 & 16.70 & 5.60 & 25.00 & 0.033 & 0.000 & 0.042 & 0.017 & 0.050 & 1.6423 & 0.7126 & 0.8238\end{array}$ $\begin{array}{lllllllllllll}8.30 & 0.00 & 8.30 & 5.60 & 8.30 & 0.008 & 0.000 & 0.017 & 0.006 & 0.017 & 1.8205 & 0.6614 & 0.8611\end{array}$ $\begin{array}{lllllllllllll}16.70 & 0.00 & 0.00 & 5.60 & 8.30 & 0.025 & 0.000 & 0.000 & 0.006 & 0.017 & 2.0852 & 0.7130 & 0.8545\end{array}$ $\begin{array}{lllllllllllll}16.70 & 0.00 & 8.30 & 11.10 & 8.30 & 0.033 & 0.000 & 0.008 & 0.022 & 0.008 & 2.2156 & 0.7596 & 0.8571\end{array}$ $\begin{array}{lllllllllllll}8.30 & 0.00 & 16.70 & 5.60 & 16.70 & 0.008 & 0.000 & 0.033 & 0.011 & 0.025 & 2.0759 & 0.8160 & 0.8889\end{array}$ $\begin{array}{lllllllllllll}33.30 & 33.30 & 16.70 & 22.20 & 25.00 & 0.033 & 0.050 & 0.008 & 0.028 & 0.025 & 2.0759 & 0.7809 & 0.8627\end{array}$ $\begin{array}{lllllllllllll}16.70 & 0.00 & 0.00 & 5.60 & 8.30 & 0.017 & 0.000 & 0.000 & 0.006 & 0.008 & 1.7299 & 0.5024 & 0.5621\end{array}$ $\begin{array}{lllllllllllll}25.00 & 0.00 & 8.30 & 11.10 & 16.70 & 0.100 & 0.000 & 0.017 & 0.061 & 0.025 & 1.4560 & 0.6405 & 0.7484\end{array}$ $\begin{array}{lllllllllllll}0.00 & 0.00 & 16.70 & 5.60 & 8.30 & 0.000 & 0.000 & 0.025 & 0.011 & 0.008 & 2.0556 & 0.6731 & 0.9048\end{array}$ $\begin{array}{lllllllllllll}8.30 & 0.00 & 0.00 & 0.00 & 8.30 & 0.008 & 0.000 & 0.000 & 0.000 & 0.008 & 1.8205 & 0.4771 & 1.0000\end{array}$ $\begin{array}{lllllllllllll}0.00 & 16.70 & 0.00 & 5.60 & 0.00 & 0.000 & 0.017 & 0.000 & 0.006 & 0.000 & 1.4427 & 0.4515 & 0.8333\end{array}$ $\begin{array}{lllllllllllll}8.30 & 0.00 & 0.00 & 0.00 & 8.30 & 0.017 & 0.000 & 0.000 & 0.000 & 0.017 & 1.0278 & 0.4686 & 0.7619\end{array}$

$\begin{array}{lllllllllllll}0.00 & 0.00 & 8.30 & 5.60 & 0.00 & 0.000 & 0.000 & 0.033 & 0.022 & 0.000 & 0.8686 & 0.4581 & 0.7111\end{array}$ $\begin{array}{lllllllllllll}0.00 & 0.00 & 8.30 & 5.60 & 0.00 & 0.000 & 0.000 & 0.025 & 0.017 & 0.000 & 1.0278 & 0.4361 & 0.7143\end{array}$ $\begin{array}{lllllllllllll}0.00 & 0.00 & 8.30 & 5.60 & 0.00 & 0.000 & 0.000 & 0.008 & 0.006 & 0.000 & 1.8640 & 0.5786 & 0.9000\end{array}$ $\begin{array}{lllllllllllll}8.30 & 0.00 & 8.30 & 5.60 & 8.30 & 0.008 & 0.000 & 0.017 & 0.006 & 0.017 & 1.6681 & 0.6142 & 0.7818\end{array}$ $\begin{array}{lllllllllllll}25.00 & 0.00 & 0.00 & 11.10 & 8.30 & 0.042 & 0.000 & 0.000 & 0.017 & 0.017 & 1.8034 & 0.7384 & 0.8583\end{array}$ $\begin{array}{lllllllllllll}0.00 & 16.70 & 16.70 & 11.10 & 8.30 & 0.000 & 0.033 & 0.033 & 0.028 & 0.008 & 1.3435 & 0.6357 & 0.7209\end{array}$ $\begin{array}{lllllllllllll}33.30 & 50.00 & 33.30 & 44.40 & 25.00 & 0.317 & 0.317 & 0.150 & 0.272 & 0.217 & 1.5822 & 0.7128 & 0.7786\end{array}$ $\begin{array}{lllllllllllll}16.70 & 16.70 & 8.30 & 11.10 & 16.70 & 0.025 & 0.033 & 0.017 & 0.017 & 0.033 & 2.5514 & 0.8591 & 0.8696\end{array}$ $\begin{array}{lllllllllllll}8.30 & 16.70 & 16.70 & 16.70 & 8.30 & 0.008 & 0.017 & 0.058 & 0.039 & 0.017 & 1.9850 & 0.7691 & 0.8182\end{array}$ $\begin{array}{lllllllllllll}16.70 & 16.70 & 33.30 & 16.70 & 33.30 & 0.017 & 0.050 & 0.092 & 0.028 & 0.092 & 1.7297 & 0.6793 & 0.6735\end{array}$ $\begin{array}{lllllllllllll}8.30 & 33.30 & 58.30 & 33.30 & 33.30 & 0.067 & 0.100 & 0.467 & 0.194 & 0.292 & 2.0011 & 0.7649 & 0.7948\end{array}$ $\begin{array}{lllllllllllll}16.70 & 16.70 & 8.30 & 5.60 & 25.00 & 0.033 & 0.033 & 0.017 & 0.011 & 0.050 & 2.3521 & 0.8937 & 0.8874\end{array}$ $\begin{array}{lllllllllllll}8.30 & 16.70 & 8.30 & 11.10 & 8.30 & 0.042 & 0.100 & 0.025 & 0.061 & 0.025 & 0.8909 & 0.4647 & 0.6330\end{array}$ $\begin{array}{lllllllllllll}8.30 & 0.00 & 0.00 & 0.00 & 8.30 & 0.008 & 0.000 & 0.000 & 0.000 & 0.008 & 0.5725 & 0.4745 & 0.6560\end{array}$ $\begin{array}{lllllllllllll}75.00 & 83.30 & 75.00 & 72.20 & 83.30 & 3.750 & 2.833 & 2.333 & 3.022 & 4.467 & 1.0418 & 0.7421 & 0.8004\end{array}$ $\begin{array}{lllllllllllll}8.30 & 0.00 & 0.00 & 5.60 & 0.00 & 0.017 & 0.000 & 0.000 & 0.011 & 0.000 & 1.6681 & 0.6923 & 0.8727\end{array}$

Cont... 
Mariscus compactus (Retz.) Druce

Scirpus grossus L.f.

Hydrocharis dubia (Blume) Backer

Limnocharis flava (L.) Buch.

Chloris barbata (L.) Sw.

Cynodon dactylon (L.) Pers.

Dactyloctenium aegyptium (L.) Beauv.

Digitaria ciliaris (Retz.) Kohler

Echinochloa colona (L.) Link.

Echinochloa crus-galli (L.) Beauv.

Eleusine indica (L.) Gaertn.

Eragrostis tenella (L.) Beauv. ex

Roem. \& Schult.

Erianthus arundinaceus (Retz.)

Jeswiel. ex Heyne

Eriochloa procera (Retz.) Hubb

Hymenachne acutigluma (Steud.)

Gillilland

Leptochloa chinensis (L.) Nees

Oryza rufipogon Griff.

Panicum repens $\mathrm{L}$.

Paspalum conjugatum Berg.

Paspalum distichum L.

Paspalum longifolium Roxb.

Paspalum notatum Flugge

Paspalum scrobiculatum L.

Pennisetum purpureum Schum.

Phragmites karka(Retz.) Trin. ex Steud.

Pseudoraphis brunoniana Griff.

Saccharum spontaneum L.

Brachiaria mutica (Forssk.) Stapf

Eichhornia crassipes (Mart.) Solms.

Monochoria elata Ridl.

Monochoria hastata (L.) Solms.

Monochoria korsakowii Regel \& Maack. $\begin{array}{lllllllllllll}16.70 & 16.70 & 8.30 & 16.70 & 8.30 & 0.017 & 0.050 & 0.017 & 0.033 & 0.008 & 2.1177 & 0.7520 & 0.8382\end{array}$ $\begin{array}{lllllllllllll}8.30 & 16.70 & 0.00 & 11.10 & 0.00 & 0.008 & 0.033 & 0.000 & 0.017 & 0.000 & 1.4427 & 0.5737 & 0.8214\end{array}$

$\begin{array}{lllllllllllll}8.30 & 0.00 & 0.00 & 5.60 & 0.00 & 0.017 & 0.000 & 0.000 & 0.011 & 0.000 & 0.9618 & 0.4515 & 0.7143\end{array}$

$\begin{array}{lllllllllllll}0.00 & 0.00 & 8.30 & 0.00 & 8.30 & 0.000 & 0.000 & 0.017 & 0.000 & 0.017 & 1.3029 & 0.5933 & 0.8222\end{array}$

$\begin{array}{lllllllllllll}16.70 & 16.70 & 0.00 & 5.60 & 16.70 & 0.025 & 0.050 & 0.000 & 0.017 & 0.025 & 1.4085 & 0.4160 & 0.4619\end{array}$

$\begin{array}{lllllllllllll}50.00 & 16.70 & 58.30 & 38.90 & 58.30 & 0.233 & 0.033 & 0.850 & 0.233 & 0.750 & 1.4318 & 0.7601 & 0.7972\end{array}$

$\begin{array}{llllllllllllll}8.30 & 0.00 & 8.30 & 5.60 & 8.30 & 0.017 & 0.000 & 0.008 & 0.011 & 0.008 & 1.5946 & 0.5495 & 0.6206\end{array}$

$\begin{array}{lllllllllllll}8.30 & 0.00 & 33.30 & 11.10 & 25.00 & 0.025 & 0.000 & 0.117 & 0.067 & 0.042 & 1.3598 & 0.6200 & 0.7104\end{array}$

$\begin{array}{lllllllllllll}58.30 & 83.30 & 83.30 & 72.20 & 75.00 & 1.200 & 1.350 & 0.833 & 0.744 & 1.592 & 1.4772 & 0.9435 & 0.8713\end{array}$

$\begin{array}{lllllllllllll}66.70 & 66.70 & 66.70 & 61.10 & 75.00 & 0.833 & 0.617 & 0.700 & 0.783 & 0.667 & 1.5966 & 0.9320 & 0.8591\end{array}$

$\begin{array}{lllllllllllll}50.00 & 33.30 & 58.30 & 38.90 & 66.70 & 0.125 & 0.217 & 0.458 & 0.194 & 0.400 & 1.3599 & 0.7686 & 0.8098\end{array}$

$\begin{array}{lllllllllllll}8.30 & 0.00 & 8.30 & 5.60 & 8.30 & 0.017 & 0.000 & 0.033 & 0.011 & 0.033 & 1.6616 & 0.7296 & 0.7958\end{array}$

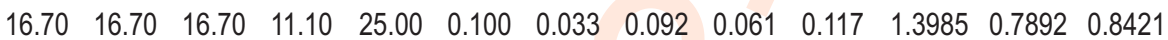

$\begin{array}{lllllllllllll}8.30 & 16.70 & 25.00 & 11.10 & 25.00 & 0.008 & 0.233 & 0.067 & 0.083 & 0.067 & 1.7631 & 0.7080 & 0.7707\end{array}$ $\begin{array}{lllllllllllll}8.30 & 0.00 & 16.70 & 5.60 & 16.70 & 0.017 & 0.000 & 0.042 & 0.011 & 0.042 & 0.7461 & 0.4954 & 0.6494\end{array}$

$\begin{array}{lllllllllllll}100.0 & 100.0 & 100.0 & 100.0 & 100.0 & 1.925 & 3.183 & 3.150 & 2.017 & 3.642 & 1.2658 & 0.9239 & 0.8627\end{array}$ $\begin{array}{lllllllllllll}75.00 & 83.30 & 66.70 & 77.80 & 66.70 & 5.150 & 2.617 & 1.442 & 5.128 & 1.458 & 1.2971 & 0.7272 & 0.7457\end{array}$ $\begin{array}{lllllllllllll}25.00 & 16.70 & 25.00 & 22.20 & 25.00 & 0.183 & 0.050 & 0.075 & 0.089 & 0.150 & 1.6368 & 0.7958 & 0.8318\end{array}$ $\begin{array}{lllllllllllll}16.70 & 0.00 & 8.30 & 5.60 & 16.70 & 0.025 & 0.000 & 0.008 & 0.011 & 0.017 & 2.2735 & 0.7675 & 0.8571\end{array}$ $\begin{array}{lllllllllllll}25.00 & 0.00 & 8.30 & 22.20 & 0.00 & 0.042 & 0.000 & 0.008 & 0.033 & 0.000 & 1.8463 & 0.6891 & 0.8095\end{array}$ $\begin{array}{lllllllllllll}8.30 & 0.00 & 8.30 & 5.60 & 8.30 & 0.008 & 0.000 & 0.017 & 0.006 & 0.017 & 2.5022 & 0.8225 & 0.9273\end{array}$ $\begin{array}{lllllllllllll}8.30 & 16.70 & 16.70 & 16.70 & 8.30 & 0.008 & 0.033 & 0.017 & 0.022 & 0.008 & 2.0121 & 0.7090 & 0.8485\end{array}$ $\begin{array}{lllllllllllll}0.00 & 0.00 & 8.30 & 5.60 & 0.00 & 0.000 & 0.000 & 0.017 & 0.011 & 0.000 & 1.5417 & 0.5871 & 0.8571\end{array}$ $\begin{array}{lllllllllllll}8.30 & 16.70 & 0.00 & 11.10 & 0.00 & 0.017 & 0.017 & 0.000 & 0.017 & 0.000 & 1.3654 & 0.5693 & 0.8056\end{array}$ $\begin{array}{lllllllllllll}0.00 & 0.00 & 8.30 & 5.60 & 0.00 & 0.000 & 0.000 & 0.025 & 0.017 & 0.000 & 0.8507 & 0.4413 & 0.5508\end{array}$ $\begin{array}{lllllllllllll}8.30 & 0.00 & 16.70 & 11.10 & 8.30 & 0.183 & 0.000 & 0.050 & 0.133 & 0.033 & 1.4595 & 0.6298 & 0.7126\end{array}$ $\begin{array}{lllllllllllll}16.70 & 0.00 & 8.30 & 16.70 & 0.00 & 0.033 & 0.000 & 0.008 & 0.028 & 0.000 & 1.1078 & 0.4244 & 0.4977\end{array}$ $\begin{array}{lllllllllllll}83.30 & 33.30 & 75.00 & 61.10 & 83.30 & 1.267 & 0.283 & 0.383 & 0.483 & 1.067 & 1.3124 & 0.7759 & 0.7912\end{array}$ $\begin{array}{lllllllllllll}0.00 & 0.00 & 33.30 & 11.10 & 16.70 & 0.000 & 0.000 & 0.117 & 0.039 & 0.058 & 1.4701 & 0.7375 & 0.8322\end{array}$ $\begin{array}{lllllllllllll}0.00 & 0.00 & 8.30 & 0.00 & 8.30 & 0.000 & 0.000 & 0.017 & 0.000 & 0.017 & 1.4427 & 0.6021 & 0.8571\end{array}$ $\begin{array}{lllllllllllll}8.30 & 0.00 & 8.30 & 0.00 & 16.70 & 0.017 & 0.000 & 0.017 & 0.000 & 0.033 & 1.3654 & 0.5529 & 0.7778\end{array}$ $\begin{array}{llllllllllllll}0.00 & 0.00 & 8.30 & 0.00 & 8.30 & 0.000 & 0.000 & 0.008 & 0.000 & 0.008 & 1.4427 & 0.3010 & 1.0000\end{array}$

C1: crop 1, C2: crop 2, C3: crop 3; In: inner dike, out: outer dike.

Table 2 : Frequency of occurrence of seasonal weed species and the environment inside and outside the dike

\begin{tabular}{llllll}
\hline Category & Group A & Group B & Group C & Group D & Group E \\
\hline Crop 1 & 66 & 16 & 10 & 4 & 5 \\
Crop 2 & 39 & 10 & 3 & 5 & 5 \\
Crop 3 & 19 & 15 & 3 & 8 & 2 \\
In dike & 77 & 23 & 6 & 5 & 1 \\
Outdike & 98 & & & & 5 \\
\hline
\end{tabular}

Table 3 : The five highest densities of rice fields surveyed in An Giang

\begin{tabular}{llllll}
\hline Species & Crop 1 & Crop 2 & Crop 3 & In dike & Out dike \\
\hline Brachiaria mutica & 1.267 & & & & 1.067 \\
Echinochloa colona & 1.200 & 1.350 & & & 1.592 \\
Leptochloa chinensis & 1.925 & 3.183 & 3.150 & 2.017 & 3.642 \\
Fimbristylis quinquangularis & 3.750 & 2.833 & 2.333 & 3.022 & 4.467 \\
Oryza rufipogon & 5.150 & 2.617 & 1.442 & 5.128 & 1.458 \\
\hline
\end{tabular}


Table 4 : Similarity index of weed species composition between crops and two sides of dike system in paddy field

\begin{tabular}{llllll}
\hline Category & Crop 1 & Crop 2 & Crop 3 & In dike & Out dike \\
\hline Crop 1 & & & & \\
Crop 2 & 79.52 & & & \\
Crop 3 & 67.39 & 67.27 & 31.25 & \\
In dike & 35.93 & 31.85 & 30.87 & 69.13 & \\
Outdike & 28.41 & 25.05 & & \\
\hline
\end{tabular}

Table 5 : Biodiversity indices of weeds collected from study sites

\begin{tabular}{llll}
\hline Category & d & H'log10) $^{\prime}$ & 1-ë \\
\hline Crop 1 & 12.8879 & 1.2039 & 0.8712 \\
Crop 2 & 8.4957 & 1.0700 & 0.8448 \\
Crop 3 & 14.7128 & 1.3448 & 0.9057 \\
Inside dike & 13.2900 & 1.2647 & 0.8895 \\
Outside dike & 13.8069 & 1.2076 & 0.8592 \\
\hline
\end{tabular}

conducted in the rice-growing area in the dike, it was not possible to grow rice outside the dike due to flooding depth of $1-4 \mathrm{~m}$, so the species composition was less diverse than in Crop 1 and Crop 3.

The number of weed species (112 species) outside thedike was more diversity than that of inside the dike (104 species); because the fields outside the dike had more aquatic plants due to the longer time the field is flooded. The typical species were found in this area including Marsilea quadrifolia, Neptunia oleracea, Hydrocera triflora, Cryptocoryne ciliata, Monochoria spp., Limnocharis flava.

The frequency of occurrence and density of weed species are detailed in Table 1. The grouping of species by frequency of occurrence is shown in Table 2.

Table 2 shows that the number of species belonging to groups C, D, E had the most in Crop 1 with 19 species, followed by Crop 2 with 12 species and Crop 3 with 11 species. Crop 1 often had hot and humid weather with heavy rain, hence, it was suitable for the growth and development of herbaceous plants. In contrast, Crop 3 experienced lack of water, drought, weeds grow poorly. The number of species with very high frequency of occurrence (Group E) was most encountered in Crop 1 with five species (Leptochloa chinensis, Alternanthera paronychioides, Ludwigia hyssopifolia, Ludwigia prostrata, Brachiaria mutica) and Crop 2 with five species (Leptochloa chinensis, Ludwigia hyssopifolia, Fimbristylis quinquangularis, Echinochloa colona, Oryza rufipogon). Crop 3 had only two species (Leptochloa chinensis and Echinochloa colona). Leptochloa chinensis especially appeared in all rice crops in the year $(\mathrm{F} \%=100 \%)$.

Table 2 shows that the number of species in groups $C, D$, $E$ in outside-dike (16 species) was higher than that in the insidedike (12 species). In particular, species belonging to group E such as Ludwigia hyssopifolia, Ludwigia prostrata, Fimbristylis quinquangularis, Leptochloa chinensis, Brachiaria mutica were mainly found in outside-dike fields. These herbs grew and developed rapidly due to availability of humidity and water.

Table 1 shows that the highest weed density (19.525 weeds $\mathrm{m}^{-2}$ ) was in Crop 1; followed by Crop 2, with an average of 16.363 weeds $\mathrm{m}^{-2}$ and the lowest is Crop 3, with an average of 14.725 weeds $\mathrm{m}^{-2}$. Due to favorable weather of Crop 1 , the density of weeds was higher than that Crops 2 and 3 . The mean density in outside-dike fields was 19.350 weeds $\mathrm{m}^{-2}$, which was higher than inside-dike fields $\left(17.228\right.$ weeds $\left.\mathrm{m}^{-2}\right)$. Five species with high individual densities of $1-6$ weeds $\mathrm{m}^{-2}$ in crops and farming environments are shown in Table 3.

Table 3 shows that three species including Leptochloa chinensis, Fimbristylis quinquangularis and Oryza rufipogon were all present in high densities in all three crops, both inside and outside the dike. These three species were also common weed species in wet rice fields in Vietnam (Suk et al., 2000).

Similarity of weed species composition among three crops and two sides of dike system in paddy field is presented in Table 4. In terms of crop, the most similar value was between crops 1 and $2(79.52 \%)$ and the least one was between Crops 2 and $3(67.27 \%)$. Likely, the flora in the inside dike was similar to that in outside with the similarity index at $69.13 \%$. The composition of weed species was not significantly different among three crops and two sides of dike system (PERMANVOA, $p>0.05)$.

The values of $d, H$ ' and $\lambda$ of three crops, and two sides of dike were high and are presented in Table 5 . Table 5 shows that the $d, H^{\prime}$ and $1-\lambda$ reached the highest point in Crop 3 and the lowest in Crop 2 when the paddy field was flooded. Consequently, 
weeds could not survive in paddy rice fields in the outer of dike system. Regarding sides of dike system, the values of indices $\mathrm{d}, \mathrm{H}$ 'and $1-\lambda$ ' differed slightly. This result shows that the dike systems had little effect on the weed species diversity between the inner and outer paddy rice fields of dike. Because in these areas, there were many canals circulating between the two rice growing areas inner and outer the dike, so spreading of weeds was easy between fields.

The weed composition in the paddy rice in An Giang was a diversity of 137 species belong to 103 genera of 42 families in 2 phyla. In which, Poaceaea, Cyperaceae and Compositae had the highest number of species. The largest number of species present were in crop 3 and in fields outside the dike system. Most weeds appear with very low frequency (Group A, F d $<20 \%$ ), only a few species appear with high frequency (Group E, F > 80\%). The highest weed density was in Crop 1. Values for the indices $d$, $H$ ' and $1-\lambda$ were highest in Crop 3. The dike system has little effect on weed composition in rice fields in An Giang province.

\section{Acknowledgments}

This study is funded in part by the Can Tho University Improvement Project VN14-P6, supported by a Japanese ODA loan.

\section{Add-on Information}

Authors' contribution : M.D. Quan: Experinment design, sample collection and classification, data analysis, manuscript writing; T.T. Hang: sample collection; S.T. Nam: data analysis, manuscript writing.

Research content : The research content of manuscript is original and has not been published elsewhere.

Ethical approval : NotApplicable

Conflict of interest : The authors declare that there is no conflict of interest.

\section{Data from other sources : NotApplicable}

Consent to publish : All authors agree to publish the paper in Journal of Environmental Biology.

\section{References}

Ban, N.T. and V.I. Dorofeev: Checklist of Plant Species of Vietnam, Vol 2. Agriculture Publishing House, Hanoi, Vietnam (2003).

Ban, N.T. and V.I. Dorofeev: Checklist of Plant Species of Vietnam, Vol 3. Agriculture Publishing House, Hanoi, Vietnam (2005).

Bray, J.R. and J.T. Curtis: An ordination of the upland forest communities of Southern Wisconsin. Ecol. Monogr., 27, 325-349 (1957).
Clarke, K.R. and R.N. Gorley: "PRIMER v6: User Manual/Tutorial,". PRIMER-E, Plymouth, United Kingdom (2006).

Dinh, Q.M., Y.T.N. Nguyen, T.H. Dang, N.S. Tran and T.T.H. Lam: The impact of human activities on the biodiversity of fish species composition in rice paddy field in An Giang Province, Southern Vietnam. Egypt. J. Aquat. Biol. Fish., 24, 107-120 (2020).

Duong, C.V., S.T.N. Tran, K.C. Le, H. Hiroyuki, T. Kazuyuki and K. Hiromi: Lowland Rice Weeds in Vietnam. Agriculture Publishing House, Ho Chi Minh City, Vietnam (2003).

Duong, Q.T., H.T.L. Tran, P.H. Pham and T.P.D. Van: Analysis of some economic and environmental aspects of agricultural cropping systems in full-dyke area, case study of Cho Moi district, An Giang province. Can Tho University Journal of Science, Thematic number: Environment and Climate Change, 1, 110-119 (2017).

Huynh, M.T., T.D.P. Van, T.H. Nguyen and M.T.V. Huynh: Impact of dike system development on rice production in An Giang province and flood dynamics on main river system in Mekong Delta. J. Meteorol. Hydrol., 02, 35-40 (2013).

Margalef, R.: Information theory in ecology. Int. J. Gen. Syst., 3, 36-71 (1958).

Nguyen, C.D. and N. Vromant: PRA - PRA - Rural Assessment with the Participation of People, Agriculture Publishing House, Ho Chi Minh City, Vietnam (2009).

Nguyen, T.N.: Plant Research Methods. Hanoi National University Press, Hanoi (2007).

Pham, D.L.M. and T.D.V. Pham: Surface water quality and self-cleaning ability of canal system in enclosed dike area in My Luong town, Cho Moi district, An Giang province. Can Tho University J. Sci., 36 , 18-26 (2015).

Nguyen, T.X., T.T. Truong, H.L.T. Tran and T.D.P. Van: Evaluate synthesize the effectiveness of the Delta flood control project Mekong river - southern Vam Nao research area. Vietnam J. Agric. Sci. Technol., 5, 95-102 (2016).

Pham, H.H.: Vietnamese Plants - An Illustrated Flora of Vietnam, Vol I. Young Publishers, Ho Chi Minh City, Vietnam (1999).

Pham, H.H.: Vietnamese Plants - An Illustrated Flora of Vietnam, Vol II. Young Publishers, Ho Chi Minh City, Vietnam (2000).

Pham, H.H.: Vietnamese Plants - An Illustrated Flora of Vietnam. Vol III, Young Publishers, Ho Chi Minh City, Vietnam (2003).

Shannon, C.E. and W. Weaver: The Mathematical Theory of Communication, University of Illinois Press, Illinois, USA (1949).

Sharma, P.D.: Ecology and Environment. Rastogi Publication, New Delhi, India (2003).

Simpson, E.H.: Measurement of diversity. Nature, 163, 688-688 (1949).

Suk, K.J., K.W. Yong, C.V. D ${ }^{\circ}$ ing and C.A. Hoang: Common weeds in Vietnam. Agriculture Publishing House, Ho Chi Minh City, Vietnam (2000).

The Plant List (2013). Version 1.1. Published on the Internet; http://www.theplantlist.org/ (Accesses: 09/07/2020).

Tran, T., N.P. Nguyen and T.L.T. Nguyen: Environmental Weeds Common in Vietnam. Ho Chi Minh City National University Publishing House, Vietnam (2018).

Vo, C.V.: Common Plant Dictionary, Vol I. Science and Technology Publishing House, Ha Noi, Vietnam (2002).

Vo, C.V.: Common Plant Dictionary, Vol II. Science and Technology Publishing House, Ha Noi, Vietnam (2004). 